\title{
Drug compliance after stroke and myocardial infarction: A comparative study
}

\author{
Hiba Arif, Bilal Aijaz, Muhammad Islam, Unber Aftab, Santosh Kumar, Saad Shafqat \\ Department of Neurology, Aga Khan University Medical College, Karachi, Pakistan
}

Background: Stroke and myocardial infarction (MI) are both life-threatening diseases of vascular origin with a tendency to recur. In both conditions, risk of recurrence is reduced through similar drug regimens. Aim: To determine if compliance with prescribed medication after stroke or MI was similar in the two populations. Setting and Design: Retrospective data collection and cross-sectional telephonic survey of patients discharged from a single academic medical center. Materials and Methods: Adult patients consecutively discharged over a two-year period with a diagnosis of first-ever stroke (ischemic or hemorrhagic) or first-ever MI (ST-elevation) were identified through ICD-9 codes. Clinical details were abstracted from hospital records. Medication compliance was assessed through a structured telephone interview. Statistical Analysis: Bivariate analysis using Chi-square and Fisher exact tests, to determine the prevalence of noncompliance in stroke versus MI patients and differences in baseline characteristics, and multivariate analysis with logistic regression to determine independent predictors of noncompliance. Results: Followup data was collected for 298 stroke and 275 MI patients. Compliance was lower in stroke patients $(68 \%$ stroke patients compliant with at least half their discharge prescriptions versus $90 \% \mathrm{Ml}$ patients; $P<0.001)$. Literacy and post-discharge follow-up were associated with greater compliance $(P<0.05$ for both). Compliance was highest with anti-hypertensive drugs ( $98 \%$ after MI, $78 \%$ after stroke), followed by anti-platelet agents (94\% after Ml, $75 \%$ after stroke) and anti-lipid agents (70\% after MI, 59\% after stroke). Patients reported simply not feeling the need, acquiring fresh medical advice or a perceived lack of benefit, as reasons for not complying with their discharge prescriptions. Conclusions: Although similar drugs are involved, compliance with prescribed regimens is appreciably lower after stroke than after MI. Our findings underscore the need for better patient education regarding secondary prevention after stroke.

Key words: Medical therapy, myocardial infarction, prevention, stroke, stroke care
Stroke and myocardial infarction (MI) continue to be leading causes of death and disability worldwide ${ }^{[1]}$ and are considered to be major public health challenges in all regions of the world. Hypertension, diabetes mellitus and elevated serum lipids are established as important risk factors for the occurrence and recurrence of both stroke and MI. ${ }^{[2-5]}$ Although prevalent in large numbers, the impact of these risk factors is modifiable, in part through medication use and in part through modifications in lifestyle. Little doubt exists regarding the efficacy of secondary prevention drug treatment following stroke and MI. ${ }^{[6-20]}$

Most available data regarding patient compliance with anticoagulant, antiplatelet and risk factor treatment is derived from controlled clinical trials. Information regarding compliance with secondary prevention for stroke and MI in routine clinical practice is scarce. Available data suggests that the public perception of the acuity and seriousness of stroke is underappreciated and that this lack of awareness may be associated with decreased compliance with secondary prevention measures and consequently a higher risk of stroke. ${ }^{[21]} \mathrm{A}$ recent study by Baune et al identified noncompliance with medication as a significant independent predictor of stroke in hypertensive patients (O.R. 6.07; 95\% C.I:1.5324.07). ${ }^{[22]}$ Stroke and MI are both life-threatening vascular insults of critical organs. Since the two conditions share common features in pathophysiology and risk-factor profile and therefore in secondary prevention strategies, it would be appropriate to compare noncompliance with pharmacological treatment in these two groups; this would enable us to specifically identify patient-related variables that may predict noncompliant behavior. We tested this hypothesis in the present study using a cross-sectional telephone survey of stroke and MI patients. Our primary objective was to determine the frequency of noncompliance in stroke and MI patients, as lagging compliance may represent an important area of educational and motivational intervention. Secondary objectives were to 
identify predictors of, and patient-reported reasons for, noncompliance in each patient group.

\section{Materials and Methods}

Primarily a cross-sectional survey, this study was conducted in two phases: (i) a retrospective medical record chart review; and (ii) a structured telephone interview. Study design was approved by the Ethics Committee at our institution. In the first phase, using ICD-9-CM (International classification of diseases, ninth revision, clinical modification) codes, hospital records were screened to identify consecutive adult (age $>18$ years) patients with first-ever stroke or first-ever MI who had been discharged during a two-year period beginning at least six months prior to the date of the search.

Stroke was defined as a focal neurological deficit of sudden onset, with a corresponding intracerebral lesion (infarct or hemorrhage) on brain imaging. ${ }^{[23]} \mathrm{MI}$ was defined as ST-segment elevation on EKG in association with derangement in cardiac enzymes. The inclusion and exclusion criteria for both groups are listed as follows: Inclusion criteria for the stroke group:

1. All patients with a stroke (as defined earlier) and CT or MRI revealing a corresponding focal intracerebral ischemic or hemorrhagic lesion and

2. Older than 18 years and residents of Karachi.

Exclusion criteria for the stroke group:

1. Patients with prior history of MI

2. Patients with a recurrent stroke

3. Presence of a nonischemic or nonhemorrhagic pathology that may have mimicked a stroke (including diagnosis of subdural or subarachnoid hemorrhage, migrainous aura or seizures)

4. Patients considered to have transient ischemic attack

Inclusion criteria for the MI group:

1. All patients with myocardial infarction of the STelevation type and

2. Older than 18 years and residents of Karachi.

Exclusion criteria for the MI group:

1. Patients with a previous history of stroke or TIA

2. Patients with a recurrent MI

In an attempt to standardize the analysis of noncompliance in these patients, we thought it appropriate to restrict our patient population to just those patients with first-ever stroke or MI. It is possible that the degree of compliance a patient shows with treatment may differ depending on whether the patient has had a similar/related prior illness. In some cases a secondary insult (MI or stroke) may serve to increase compliance while for others, compliance to treatment may become worse as the patient develops feelings of helplessness and starts doubting the use of medication in the face of recurrent vascular events.
This may be especially true in cases where regular followup is not obtained and the need to continue medication is not adequately stressed by a physician/caregiver.

Clinical details of the index stroke or MI were abstracted from the hospital record. Details regarding demographic variables, comorbid conditions, medications prescribed at discharge after the index stroke or MI and length of stay were obtained. Patient compliance was categorized as poor (0-25\%), low (26-50\%), moderate (51-75\%) or high (76-100\%), based on the percentage of prescribed medication being consumed as directed.

Potential subjects were then contacted via telephone and using a structured questionnaire, we assessed drug compliance, reasons for noncompliance (if any) to treatment prescribed after the index stroke or MI, current level of disability and education attained. The questionnaire had been pilot-tested in ten patients (five with MI and five with stroke) and adjusted accordingly. To assess education, the highest level of schooling obtained by each subject was recorded (primary or secondary school, matriculation, bachelors or postgraduation). However, since the number of patients in each of these subcategories was small, for the purpose of the analysis, all subjects who had ever gone to school were counted as "literate" and collectively analyzed as a predictor of noncompliance. If patients were unavailable or unable to communicate, information was obtained from the primary caregiver. Patients who could not be reached despite three telephone attempts were not pursued further. Informed consent was obtained prior to each interview.

\section{Statistical analysis}

Descriptive statistics (frequencies) and bivariate analyses (Chi-square and Fisher exact tests, as appropriate) were employed to analyze noncompliance in stroke versus MI patients and differences in baseline characteristics; multivariate analysis with forward conditional logistic regression was used to identify independent predictors of noncompliance. Variables tested as potential predictors included demographic variables (age, gender, level of education), comorbid illness (hypertension, diabetes and dyslipidemia) and hospital length of stay.

\section{Results}

\section{Study population}

During the study period, 603 patients (325 with stroke, 278 with MI) matched our inclusion criteria, of which 573 (95\%) were available for telephone interview. Of the 30 patients who could not be interviewed, 27 had stroke (18 expired, six inaccessible by telephone, three refused consent) and three had MI (all inaccessible by telephone). Thus follow-up data was finally collected on 298 patients with stroke and 275 patients with MI. Table 1 presents their baseline characteristics. 


\section{Compliance in stroke vs. MI patients}

Patient compliance was categorized as poor $(0-25 \%)$, low (26-50\%), moderate (51-75\%) or high (76-100\%), based on the percentage of prescribed medication being consumed as directed. High compliance was seen in 179 of 298 (60\%) stroke patients, compared with 205 of 275 (75\%) MI patients. Of the remaining stroke patients, $8 \%$ had moderate compliance, $20 \%$ were low and $12 \%$ poor. Of the remaining MI patients, $15 \%$ had moderate compliance, $8 \%$ were low and only $2 \%$ poor. Thus, up to $90 \%$ of MI patients were consuming at least half of their prescription, compared with $68 \%$ stroke patients. Overall, stroke patients were significantly less compliant than MI patients $[P<0.001$ across all categories of compliance; Table 2].

Defining "adequate" compliance as consumption of $>$ $50 \%$ of the prescribed regimen, we found that adequately compliant patients in both groups were more likely to be literate and more likely to report regular post-discharge follow-up, compared with noncompliant patients $[P<0.001$ for both; Table 3]. Additionally, patients with dyslipidemia, though not with other co-morbidities, were more likely to be adequately compliant $(P<0.001)$. As the number of co-morbid conditions and discharge medications increased, a trend towards noncompliant behavior was noted in both the stroke and MI groups though the difference was not statistically significant (results not shown).

\section{Compliance with types of medication}

We also examined adequate compliance with particular types of medication, including anti-platelet agents, anticoagulants and risk-factor-modifying drugs such as antihypertensive, antidiabetic and lipid-lowering agents [Table 4]. In both the stroke and MI groups, the highest compliance was noted for antihypertensives $(78 \%$ in stroke group, $98 \%$ in MI group; $P<0.001$ ), followed by antiplatelet agents (75\% in stroke group, $94 \%$ in MI group; $P<0.001$ ) and lipid-lowering agents (59\% in stroke group, $70 \%$ in MI group; $P<0.001)$. The lowest rates of compliance were noted for antidiabetics (28\% and $22 \%$ in the stroke and MI groups, respectively) and anticoagulants (7\% and 5\%); differences across the stroke and MI groups for these medication types were not statistically significant.

\section{Reasons cited for noncompliance}

Patients (or their surrogates) reporting inadequate compliance (consumption of $<50 \%$ of prescribed regimen) were questioned about the reasons behind discontinuing prescribed medication [Table 5]. Respondents were asked to select all applicable reasons from the following multiplechoice selection: lack of clinical improvement; did not feel the need; received new medical advice; found it too expensive; found it too inconvenient; could not tolerate side-effects; could not find it in the market; or other. In both the stroke and MI groups, respondents reported that they "did not feel the need," as the commonest reason for not complying with both anti-platelet and lipid-lowering therapy. For anticoagulants, the commonest reason for discontinuation was "new medical advice." For antidiabetic medication, "no need felt" and "new medical

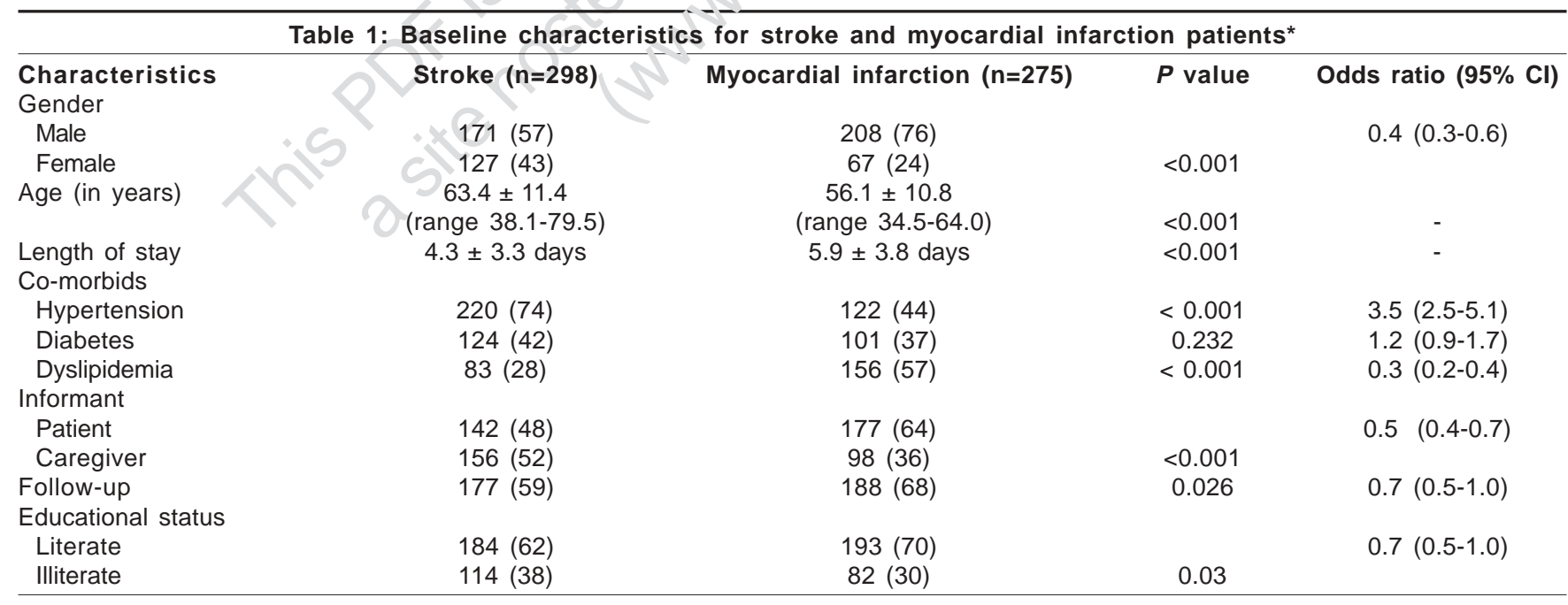

${ }^{*}$ Results are presented as number (percentage) and mean \pm standard deviation.

Table 2: Prevalence of compliance by range (\%) in stroke and myocardial infarction patients

\begin{tabular}{|c|c|c|c|c|}
\hline Compliance range (\%) & Stroke $(n=298)$ & Myocardial infarction $(n=275)$ & $P$-value & Odds ratio $(95 \% \mathrm{Cl})$ \\
\hline $0-25$ & $35(12)$ & $6(2)$ & & 1 \\
\hline $26-50$ & $59(20)$ & $23(8)$ & $<0.001$ & $2.3(0.8-6.1)$ \\
\hline $51-75$ & $25(8)$ & $41(15)$ & & $0.6(3.5-26.0)$ \\
\hline $76-100$ & $179(60)$ & $205(75)$ & & $6.7(2.7-16.3)$ \\
\hline
\end{tabular}


Table 3: Prevalence of noncompliance for stroke and myocardial infarction patients

\begin{tabular}{|c|c|c|c|c|c|c|c|c|}
\hline \multicolumn{5}{|c|}{ Stroke } & \multicolumn{4}{|c|}{ Myocardial infarction } \\
\hline Characteristics & $\begin{array}{c}\text { Compliance } \\
(n=204)\end{array}$ & $\begin{array}{c}\text { Non-compliance } \\
(\mathrm{n}=94)\end{array}$ & $P$-value & $\begin{array}{l}\text { Odds ratio } \\
(95 \% \mathrm{Cl})\end{array}$ & $\begin{array}{c}\text { Compliance } \\
(n=247)\end{array}$ & $\begin{array}{c}\text { Non-compliance } \\
(\mathrm{n}=28)\end{array}$ & $P$-value & $\begin{array}{l}\text { Odds ratio } \\
(95 \% \mathrm{Cl})\end{array}$ \\
\hline \multicolumn{9}{|l|}{ Gender } \\
\hline Male & $117(57)$ & $54(57)$ & & 1.0 & $184(79)$ & $24(87)$ & & 0.5 \\
\hline Female & $87(43)$ & $40(43)$ & 0.988 & $(0.6-1.7)$ & $63(77)$ & $4(14)$ & 0.190 & $(0.1-1.6)$ \\
\hline Age (years) & $62.6 \pm 12.1$ & $65.0 \pm 9.7$ & 0.071 & - & $55.9 \pm 10.7$ & $56.7 \pm 11.3$ & 0.734 & - \\
\hline $\begin{array}{l}\text { Length of stay } \\
\text { (days) }\end{array}$ & $4.3 \pm 4.5$ & $4.3 \pm 3.8$ & 0.995 & - & $5.9 \pm 3.8$ & $6.3 \pm 4.1$ & 0.602 & - \\
\hline \multicolumn{9}{|l|}{ Co-morbids } \\
\hline Hypertension & $157(77)$ & $63(67)$ & 0.070 & $1.6(0.9-2.9)$ & $111(50)$ & $11(39)$ & 0.568 & $1.3(0.5-3.0)$ \\
\hline Diabetes & $91(45)$ & $33(35)$ & 0.122 & $1.5(0.9-2.6)$ & $91(37)$ & $10(36)$ & 0.907 & $1.1(0.4-2.6)$ \\
\hline Dyslipidemia & $65(32)$ & 18 (19) & 0.023 & $2.0(1.0-3.7)$ & $207(46)$ & $7(25)$ & $<0.001$ & $15.5(5.8-43.4)$ \\
\hline \multicolumn{9}{|l|}{ Informant } \\
\hline Patient & $196(52)$ & $36(38)$ & & 1.7 & $161(65)$ & $16(57)$ & & $1.4(0.6-3.3)$ \\
\hline Caregiver & $98(48)$ & $58(62)$ & 0.028 & $(1.0-3.0)$ & $86(35)$ & $12(43)$ & 0.400 & \\
\hline Follow-up & $153(75)$ & $24(26)$ & $<0.001$ & $8.8(4.8-16.0)$ & $185(75)$ & $3(11)$ & $<0.001$ & $24.9(6.8-107.3)$ \\
\hline \multicolumn{9}{|c|}{ Educational status } \\
\hline Literate & $137(67)$ & $47(50)$ & 0.005 & 2.0 & $178(72)$ & $15(54)$ & & 2.2 \\
\hline Illiterate & $67(33)$ & $47(50)$ & & $(1.2-3.5)$ & $247(28)$ & $13(46)$ & 0.043 & $(0.9-5.3)$ \\
\hline
\end{tabular}

Table 4: Compliance with various drug categories

\begin{tabular}{|c|c|c|c|c|c|c|c|c|}
\hline \multirow[b]{2}{*}{ Drug category } & \multirow[b]{2}{*}{$\begin{array}{c}\text { Compliance } \\
(n=204)\end{array}$} & \multicolumn{3}{|c|}{ Stroke } & \multicolumn{4}{|c|}{ Myocardial infarction } \\
\hline & & $\begin{array}{c}\text { Non-compliance } \\
(n=94)\end{array}$ & $P$-value & $\begin{array}{c}\text { Odds ratio } \\
(95 \% \mathrm{Cl})\end{array}$ & $\begin{array}{c}\text { Compliance } \\
(n=247)\end{array}$ & $\begin{array}{c}\text { Non-compliance } \\
(n=28)\end{array}$ & $P$-value & $\begin{array}{l}\text { Odds ratio } \\
(95 \% \mathrm{Cl})\end{array}$ \\
\hline Antiplatelets & $153(75)$ & $11(12)$ & $<0.001$ & $22.6(10.7-48.9)$ & $233(94)$ & - & *- & - \\
\hline Anticoagulants & $15(7)$ & - & *- & $\infty-1$ & $13(5)$ & - & $*_{-}$ & - \\
\hline Antidiabetics & $58(28)$ & $19(20)$ & 0.132 & $1.6(0.8-3.0)$ & $54(22)$ & $5(18)$ & 0.625 & $1.3(0.4-4.1)$ \\
\hline Antihypertensive & e $158(78)$ & $44(47)$ & $<0.001$ & $3.9(2.2-6.8)$ & $242(98)$ & $23(82)$ & 0.001 & $10.5(2.4-46.2)$ \\
\hline Antilipemics & $121(59)$ & $2(2)$ & $<0.001$ & $67.1(15.7-404.8$ & $174(70)$ & - & *_- & - \\
\hline
\end{tabular}

${ }^{*} P$-value statistically invalid due to zero patients in comparison group

advice" were cited with equal frequency as reasons for noncompliance. For anti-hypertensive medication, the leading reasons for noncompliance were "no need felt" (MI group) and "new medical advice" (stroke group)."

Multiple logistic regression showed that illiteracy and the presence of dyslipidemia as a comorbid condition were independent predictors of noncompliance with medication in the stroke group; in the MI group, female gender and illiteracy were predictive of noncompliance [Table 6].

\section{Discussion}

Detailed knowledge about long-term compliance with secondary stroke prevention measures is necessary to reduce rates of stroke recurrence, improve the care of stroke patients and develop more effective strategies to tackle the population burden of stroke. Judging against post-MI drug compliance, we found that post-stroke drug compliance falls significantly short across multiple categories. There are several reasons why post-MI compliance is an important benchmark in this comparison. Both ischemic stroke and myocardial infarction share pathophysiologic similarities; they are associated with similar risk factors; and their recurrence is prevented by similar measures, including the same types of medication. Moreover, MI as a disease concept carries a significant and serious public image reflecting urgency and the possibility of devastating consequences, including functional impairment and death - an image that stroke equally deserves but appears not to have achieved. We noted several differences in baseline characteristics

\begin{tabular}{|c|c|c|c|c|}
\hline $\begin{array}{c}\text { Antiplatelets } \\
\text { n (\%) }\end{array}$ & $\begin{array}{c}\text { Anticoagulants } \\
\mathrm{n}(\%)\end{array}$ & $\begin{array}{c}\text { Antidiabetics } \\
\text { n (\%) }\end{array}$ & $\begin{array}{c}\text { Antihypertensives } \\
\text { n (\%) }\end{array}$ & $\begin{array}{c}\text { Antilipidemics } \\
\text { n (\%) }\end{array}$ \\
\hline \multicolumn{5}{|c|}{ troke } \\
\hline $42(31.3)$ & $2(0.7)$ & $2(0.9)$ & $5(5.2)$ & $42(24)$ \\
\hline $8(6)$ & $9(3.2)$ & $2(0.9)$ & $10(10.4)$ & $14(8)$ \\
\hline \multicolumn{5}{|c|}{ Ayocardial infarction } \\
\hline $27(64.3)$ & $2(0.8)$ & - & $3(30)$ & $30(29.1)$ \\
\hline $1(2.4)$ & $6(2.3)$ & - & $2(20)$ & - \\
\hline $5(11.9)$ & - & - & $2(20)$ & $18(17.5)$ \\
\hline
\end{tabular}


Table 6: Independent risk factors of noncompliance for stroke and myocardial infarction patients identified by multiple logistic regression

\begin{tabular}{|c|c|c|c|}
\hline $\begin{array}{l}\text { Characteristics } \\
\text { Stroke } \\
\text { Dyslipidemia }\end{array}$ & Adjusted odds ratio & $95 \%$ confidence interval for odds ratio & $P$-value \\
\hline No & 1 & & \\
\hline Yes & 0.5 & $(0.3-0.9)$ & 0.018 \\
\hline \multicolumn{4}{|l|}{ Educational status } \\
\hline Literate & 1 & & \\
\hline Illiterate & 2.1 & $(1.3-3.5)$ & 0.004 \\
\hline \multicolumn{4}{|c|}{ Myocardial infarction } \\
\hline Male & 1 & & \\
\hline Female & 3.8 & $(1.2-12.3)$ & 0.028 \\
\hline \multicolumn{4}{|l|}{ Educational status } \\
\hline Literate & 1 & & \\
\hline Illiterate & 3.5 & $(1.5-8.3)$ & 0.005 \\
\hline
\end{tabular}

between stroke and MI patients. As our data is based on hospitalization records, this may reflect selection or access bias that could disappear in population-based comparisons. Alternately, as stroke and MI are separate pathologic entities, these observations may represent true differences between distinct disease populations. Additional studies are needed to explore this question further.

Variations in social norms or self-perception may be responsible for the differences we have observed. Stroke patients may be more resigned towards their condition, accepting their morbidity as an unavoidable part of old age. This may, in turn, be responsible for infrequent follow-up visits in the stroke group. On the other hand, Sappok et al reported higher compliance rates in older patients, ${ }^{[24]}$ while another survey showed that primary care is mainly targeted towards patients who have a high absolute risk of stroke recurrence. ${ }^{[25]}$

Compared to MI patients, there is a higher level of physical handicap in patients after stroke, which has an independent effect on the occurrence of post-stroke depression (PSD). A distinct clinical entity, PSD has a one-year cumulative incidence approaching 39\%. ${ }^{[26]} \mathrm{A}$ study by Sugisawa et $a I^{[27]}$ showed that among stroke patients subjective well-being and compliance correlates inversely with the degree of depression and impairment in activities of daily living and if three years or more have elapsed since the stroke.

Stroke is associated with painless onset and treatment delays. Nondominant hemispheric strokes, moreover, are characterized by anosognosia, which may compound the delay in seeking treatment. In contrast, MI patients may be motivated to comply with secondary prevention in order not to relive the pain and discomfort of the index episode. Community awareness may also play a role. Samsa et al showed that only $41 \%$ of individuals at increased risk for stroke were actually aware of their higher risk. ${ }^{[28]}$ This figure is in contrast to results from the REACT study, ${ }^{[29]}$ which revealed that knowledge of chest pain as an important heart attack symptom is high in the general population (89.7\%). Additionally, in our study, a significantly greater proportion of stroke patients were illiterate as compared to MI patients and illiteracy emerged as a predictor of noncompliance.

In the stroke group, patients with dyslipidemia were more likely to be compliant with medication than those without dyslipidemia. This may be a chance observation that will disappear in a larger sample. On the other hand, this observation may truly reflect increased awareness about the importance of medication use in dyslipidemic patients, who may have received reinforcement on this issue from their physicians or other care providers. Simply knowing a target lipid figure that can be lowered through drug use may also account for greater compliance and self-awareness.

Female gender was also found to be predictive of noncompliance. In our population, the reasons for this may have a socio-cultural basis. Women in Pakistan have limited access to healthcare and the health of men (as earning members of the household) is consistently prioritized. For women, this may mean fewer encounters with physicians (to enable reinforcement of the need for regular medication use) and a deteriorating sense of wellbeing, regardless of functional disability. This was also reported in a recent study by Chong et al, ${ }^{[30]}$ who found that among those in the highest functional category, women (with stroke) were more likely to report incomplete recovery and greater need for help than men. Among these women, depressed mood was associated with a response of a need for help despite a good functional recovery. Clearly, patient perception of outcomes after an event such as stroke or MI may differ between men and women and the confounding effects of depression may need to be taken into account in future studies and in clinical practice.

In analyzing compliance by type of drug we found that in both disease groups, the highest compliance rates were for antihypertensive therapy. Previously, compliance rates as high as $90.8 \%$ have been reported for antihypertensive drugs. ${ }^{[24]}$ This may be attributable to heightened public awareness about hypertension and its consequences; existence of an objective and visible target such as a 
numerical blood pressure figure may also be a factor. Somewhat lower compliance figures were seen with antiplatelet and antilipid drugs. The commonest explanation cited by patients for discontinuing these medications is that they simply did not feel the need. Compliance with anticoagulants was low, which is at least partly explained by the constraints imposed by regular physician-patient contact and monitoring.

Our results point to an important area where stroke prevention efforts fail to measure up. The lower drug compliance in stroke patients is unlikely to be due to environmental or social factors particular to a developing country like Pakistan, as the comparison group of MI patients was drawn from the same community and healthcare facility. The need to elevate awareness regarding the importance and efficacy of secondary stroke prevention at several levels is underscored. This can be done through targeted educational interventions at the individual patient level (e.g., at discharge and follow-up visits), but individual efforts must be complemented by CME for enhancing physician awareness as well as mass education to accentuate the image of cerebrovascular events as a serious public health concern at par with coronary events.

\section{References}

1. Murray CJ, Lopez AD. Mortality by cause for eight regions of the world: Global Burden of Disease Study. Lancet 1997;349:1269-76.

2. Ong TZ, Raymond AA. Risk factors for stroke and predictors of one-month mortality. Singapore Med J 2002;43;517-21.

3. Hyman DJ, Pavlik VN. Uncontrolled hypertension as a risk for coronary artery disease: Patient characteristics and the role of physician intervention. Curr Atheroscler Rep 2003;5:131-8.

4. Kaur J, Singh P, Sowers JR. Diabetes and cardiovascular diseases. Am J Ther 2002;9:510-5.

5. Summary of the second report of the National Cholesterol Education Program (NCEP) Expert Panel on Detection, Evaluation, and Treatment of High Blood Cholesterol in Adults (Adult Treatment Panel II). JAMA 1993;269:3015-23.

6. Hart RG, Benavente O, McBride R, Pearce LA. Antithrombotic therapy to prevent stroke in patients with atrial fibrillation: A meta-analysis. Ann Intern Med 1999;131:492-501.

7. Post-stroke antihypertensive treatment study. A preliminary result. PATS Collaborating Group. Chin Med J Engl 1995;108:710-7.

8. Stroke prevention by the practitioner. Cerebrovasc Dis 1999;9:1-70.

9. Albers GW, Easton JD, Saceo RL, Teal P. Antithrombotic and thrombolytic therapy for ischemic stroke. Chest 1998;114:683S-98S.

10. Du X, Cruickshank K, McNamee R, Saraee M, Sourbutts J, Summers $A$, et al. Case-control study of stroke and the quality of hypertension control in north west England. BMJ 1997;314:272-6.

11. Gueyffier F, Boissel JP, Boutitie F, Pocock S, Coope J, Cutler J, et $a l$. Effect of antihypertensive treatment in patients having already suffered from stroke. Gathering the evidence. The INDANA (Individual Data Analysis of Antihypertensive intervention trials) Project Collaborators. Stroke 1997;28:2557-62.
12. Sacco RL, Wolf PA, Gorelick PB. Risk factors and their management for stroke prevention: Outlook for 1999 and beyond. Neurology 1999;53:S15-24

13. Cassell J. Evidence-based medicine and treatment choices. Lancet $1997 ; 349: 570$.

14. Wannamethee SG, Shaper AG, Whincup PH, Walker M. Smoking cessation and the risk of stroke in middle-aged men. JAMA $1995 ; 274: 155-60$

15. PROGRESS Collaborative Group. Randomized trial of a perindopril-based blood-pressure-lowering regimen among 6,105 individuals with previous stroke or transient ischaemic attack. Lancet 2001;358:1033-41.

16. Callahan A. Cerebrovascular disease and statins: A potential addition to the therapeutic armamentarium for stroke prevention. Am J Cardiol 2001;88:33J-7J.

17. Collins R, Peto R, Armitage J. The MRC/BHF Heart Protection Study: Preliminary results. Int J Clin Pract 2002;56:53-6.

18. Jackson R, Stewart A, Beaglehole R. Trends in coronary heart disease mortality and morbidity in Auckland, New Zealand, 19741986. Int J Epidemiol 1990;19:279-83.

19. Sigfusson N, Sigvaldason H, Steingrimsdottir L, Gudmundsdottir II, Stefansdottir I, Thorsteinsson T, et al. Decline in ischaemic heart disease in Iceland and change in risk factor levels. BMJ 1991;302:1371-5

20. Vartiainen E, Puska P, Pekkanen J, Tuomilehto J, Jousilahti P. Changes in risk factors explain changes in mortality from ischaemic heart disease in Finland. BMJ 1994;309:23-7.

21. Karttunen V, Alfthan G, Hiltunen L, Rasi V, Kervinen K, Kesaniemi YA, et al. Risk factors for cryptogenic ischaemic stroke. Eur J Neurol 2002;9:625-32.

22. Baune BT, Aljeesh Y, Bender R. Factors of non-compliance with the therapeutic regimen among hypertensive men and women: A case-control study to investigate risk factors of stroke. Eur J Epidemiol 2005;20:411-9.

23. Shafqat S, Kvedar JC, Guanci MM, Chang Y, Schwamm LH. Role for telemedicine in acute stroke. Feasibility and reliability of remote administration of the NIH stroke scale. Stroke 1999;30:2141-5.

24. Sappok T, Faulstich A, Stuckert E, Kruck H, Marx P, Koennecke HC. Compliance with secondary prevention of ischemic stroke: A prospective evaluation. Stroke 2001;32:1884-9.

25. Coppola WG, Whincup PH, Walker M, Ebrahim S. Identification and management of stroke risk in older people: A national survey of current practice in primary care. J Hum Hypertens 1997;11:185-91.

26. Aben I, Denollet J, Lousberg R, Verhey F, Wojciechowski F, Honig A. Personality and vulnerability to depression in stroke patients: A 1-year prospective follow-up study. Stroke 2002;33:2391-5.

27. Sugisawa H. Treatment compliance and subjective well-being among post-stroke patients. Nippon Koshu Eisei Zasshi 1991;38:70-8.

28. Samsa GP, Cohen SJ, Goldstein LB, Bonito AJ, Duncan PW, Enarson C, et al. Knowledge of risk among patients at increased risk for stroke. Stroke 1997;28:916-21.

29. Goff DC Jr, Sellers DE, McGovern PG, Meischke H, Goldberg RJ, Bittner V, et al. Knowledge of heart attack symptoms in a population survey in the United States: The REACT Trial. Rapid Early Action for Coronary Treatment. Arch Intern Med 1998;158:2329-38.

30. Chong JY, Lee HS, Boden-Albala B, Paik MC, Saceo RL. Gender differences in self-report of recovery after stroke: The Northern Manhattan Study. Neurology 2006;67:1282-4.

Accepted on 22-01-2007

Source of Support: Funded in part by an Aga Khan University Seed Grant to Dr. Shafqat, Conflict of Interest: None declared. 